Instituto Internacional de Investigación y Desarrollo Tecnológico Educativo INDTEC, C.A.

DOI: https://doi.org/10.29394/Scientific.issn.2542-2987.2021.6.21.7.144-162

OAI-PMH: http://www.indteca.com/ojs/index.php/Revista Scientific/oai

Artículo Original / Original Article

\title{
Síndrome de Burnout en personal de salud que brinda atención a pacientes con COVID-19
}

\author{
Autores: Edwin Guillermo Valencia González \\ Universidad Tecnológica Indoamérica, UTI \\ edwinvalencia@uti.edu.ec \\ Quito, Ecuador \\ https://orcid.org/0000-0002-9631-3006 \\ Daniela Alejandra Correa Sinche \\ Universidad Tecnológica Indoamérica, UTI \\ danny ale0202@hotmail.com \\ Quito, Ecuador \\ https://orcid.org/0000-0002-6848-916X \\ Vanesa Katherine Pazmiño Cruz \\ Universidad Tecnológica Indoamérica, UTI \\ vanepazzmi1998@hotmail.es \\ Quito, Ecuador \\ https://orcid.org/0000-0003-3805-6408
}

\begin{abstract}
Resumen
El síndrome de Burnout presenta tres dimensiones (agotamiento emocional, despersonalización y realización personal), caracterizado por ser una alteración generada por un desgaste a nivel físico, mental y emocional debido a situaciones estresantes o exigencias sociales, laborales y personales excesivas, esto ha aumentado debido al COVID-19. EI objetivo de la presente investigación es estimar los niveles de prevalencia del síndrome de Burnout en el personal de salud que atiende a pacientes con COVID-19; dicha investigación es de método cuantitativo con nivel descriptivo, presenta una sola variable de diseño no experimental tipo transversal; en cuanto a la recolección de información se utilizó una encuesta de datos sociodemográficos y el Inventario de Burnout de Maslach (MBI). Los participantes fueron 40 profesionales del área de salud, en donde se presenta indicios de Burnout en una dimensión con el $32,50 \%$ y en dos dimensiones con el $30,00 \%$, siendo la dimensión de despersonalización la de mayor prevalencia con el 57,5\%; por el contrario, el 37,50\% restante no presenta afectación en ninguna dimensión. En el análisis parcial la mayor afectación se da en mujeres en un $62,5 \%$, rango de edades entre 31 a 40 años con el $45 \%$, casados en $50,0 \%$, que viven con familia $80,0 \%$ y que tienen hijos $80,0 \%$.
\end{abstract}

Palabras clave: COVID-19; desgaste; pandemia; síndrome de burnout. Código de clasificación internacional: 6109.05 - Comportamiento en la organización.

Cómo citar este artículo:

Valencia, E., Correa, D., \& Pazmiño, V. (2021). Síndrome de Burnout en personal de salud que brinda atención a pacientes con COVID-19. Revista Scientific, 6(21), 144-162, e-ISSN: 2542-2987. Recuperado de: https://doi.org/10.29394/Scientific.issn.2542-2987.2021.6.21.7.144-162

Fecha de Recepción: 20-03-2021
Fecha de Aceptación:

07-07-2021
Fecha de Publicación: 05-08-2021 
OAl-PMH: http://www.indteca.com/ojs/index.php/Revista Scientific/oai

Artículo Original / Original Article

\title{
Burnout syndrome in health personnel who provide care to patients with COVID-19
}

\begin{abstract}
The Burnout syndrome has three dimensions (emotional exhaustion, depersonalization and personal fulfillment), characterized by being an alteration generated by physical, mental and emotional wear due to stressful situations or excessive social, work and personal demands, this has increased due to to COVID-19. The objective of this research is to estimate the prevalence levels of Burnout syndrome in health personnel who care for patients with COVID-19; Said research is of a quantitative method with a descriptive level, it presents a single variable of a non-experimental crosssectional design; Regarding the collection of information, a survey of sociodemographic data and the Maslach Burnout Inventory (MBI) were used. The participants were 40 professionals from the health area, where signs of Burnout are presented in one dimension with $32.50 \%$ and in two dimensions with $30.00 \%$, with the dimension of depersonalization being the one with the highest prevalence with $57,5 \%$; on the contrary, the remaining $37.50 \%$ did not show affectation in any dimension. In the partial analysis, the greatest affectation occurs in women in $62.5 \%$, age range between 31 and 40 years with $45 \%$, married in $50.0 \%$, living with family $80,0 \%$ and having children $80,0 \%$.
\end{abstract}

Keywords: COVID-19; wear; pandemic; burnout syndrome. International classification code: 6109.05 - Organizational behaviour.

How to cite this article:

Valencia, E., Correa, D., \& Pazmiño, V. (2021). Burnout syndrome in health personnel who provide care to patients with COVID-19. Revista Scientific, 6(21), 144-162, e-ISSN: 2542-2987. Recovered from: https://doi.org/10.29394/Scientific.issn.2542-2987.2021.6.21.7.144-162

Date Received: 20-03-2021
Date Acceptance: 07-07-2021
Date Publication: 05-08-2021 


\section{Introducción}

El psiquiatra norteamericano Herbert Freudenberger (1926-1999) utilizó por primera vez el término Burnout en el año 1974, para describir el estado físico y mental de jóvenes voluntarios que trabajaban en contacto con personas en una clínica situada en Nueva York, Estados Unidos; los mismos se esforzaban en sus funciones para cumplir con las expectativas de sus superiores, después de tres años presentaron conductas de irritación, agotamiento, actitudes de cinismo con clientes y tendencia a evitarlos, por lo que se desarrolló el concepto de "estar quemado" para hacer referencia a este síndrome.

En este mismo orden de ideas, Maslach (1976), citada por Martínez (2010): propuso que el término Burnout solo sea aplicable para labores en donde se trabaje en contacto directo con personas dentro de una organización, sin embargo, cuando este síndrome tomó relevancia a nivel mundial, Herbert Freudenberger y Jeffrey Talbot Richelson (1949-2017), en 1980 lo detallaron como un estado de frustración que se genera por la dedicación a un objetivo específico sin recibir una recompensa. Finalmente, Maslach y Jackson (1981): lo definieron como un síndrome el cual está compuesto por tres dimensiones empezando por agotamiento o cansancio emocional, despersonalización y baja realización personal.

En la actualidad el síndrome de Burnout se caracteriza por ser una enfermedad o alteración generada por un desgaste a nivel físico, mental y emocional debido a situaciones estresantes o exigencias excesivas tanto sociales, laborales y personales que ocurren en el diario vivir; el mismo está compuesto por las siguientes dimensiones: 1. Agotamiento emocional, la misma hace referencia al estado afectivo y emocional; 2. Despersonalización, tiene hincapié en reacciones negativas; y 3 . Realización personal, baja satisfacción de avance en su trabajo.

El Departamento de Asistencia Técnica para la Prevención de Riesgos 
Laborales (UGT-Andalucía, 2009): menciona que este síndrome atraviesa una serie de fases importantes: en la primera fase de entusiasmo hay niveles altos de expectativas; la segunda fase se caracteriza por el descontento debido al incumplimiento de sus expectativas asociado al esfuerzo realizado; la tercera fase está asociada con la frustración lo cual genera irritabilidad y conflictos, en la cuarta se producen cambios conductuales generando daño a las personas de su entorno y afectando su desempeño laboral; finalmente la fase de quemado, en donde hay un colapso cognitivo/emocional es decir una paralización o disminución de las actividades.

Para evaluar las distintas dimensiones y fases del síndrome de Burnout la escala más utilizada y aceptada en la actualidad es el instrumento Maslach Burnout Inventory (MBI), elaborado por Maslach, Jackson y Leiter (1996): la misma tiene tres revisiones del ámbito científico y su objetivo es evaluar distintos factores del desgaste profesional, la utilización del mismo ha permitido crear estrategias y analizar el impacto en los distintos ámbitos en los que se desarrollan las personas cotidianamente.

Existe situaciones sociales que pueden profundizar la aparición de este síndrome en mayor o menor medida, una de estas situaciones se ha presentado debido a la pandemia actual a nivel mundial que ha logrado causar crisis sociales y afectaciones a toda la población en general siendo las más vulnerables las que se encuentran involucradas al trabajo en el sistema sanitario ya que el mismo ha sido desabastecido llegando al límite de su capacidad; se ha demostrado mediante diversas investigaciones que el personal de salud tiene mayor riesgo para desarrollar este síndrome.

Para Juárez (2020): como para muchos la pandemia se ha convertido en uno de los retos más grandes a nivel mundial para la salud, economía y sobre todo para la salud mental debido a la grave situación actual, aunque Álvarez (2020): refiere que la rápida propagación con la que se transmite el nuevo coronavirus no es diferente a otros virus que han desencadenado 
epidemias o pandemias, lo que cambió fue la velocidad de procesamiento de la información y la manera de percibir las cosas, ya que durante el tiempo que el virus ha estado presente ha producido a nivel mundial grandes retos, categorizándolo como una de las más grandes pandemias del último siglo.

De acuerdo con Velázquez (2020): este nuevo virus conocido como SARS-CoV-2 o COVID-19 deja grandes huellas por tantas vidas perdidas, repercusiones psicológicas, incertidumbre, entre otras consecuencias, las cuales definen una manera de afrontar el nuevo presente y futuro, ya que ha provocado hasta el año 2020 aproximadamente 1,8 millones de muertos de toda edad, sin embargo las personas más vulnerables y con mayor riesgo de contagio son las que padecen enfermedades como hipertensión arterial, diabetes, cáncer, entre otras.

La Organización Mundial de la Salud (OMS, 2020): hace referencia a que este virus no va a desaparecer, por lo que los seres humanos tendrán que enfrentar de la mejor manera la nueva realidad en la que están inmersos y las secuelas que deja la pandemia del COVID-19 debido a distintas situaciones como cuarentenas, confinamientos y restricciones como menciona Espada, et al. (2020), citados por Balluerka, Gómez, Hidalgo, Gorostiaga, Espada, Padilla y Santed (2020a):

Podemos esperar dos tipos de situaciones de afectación psicológica que pueden darse durante y tras el confinamiento [...] casos de personas con alteraciones emocionales o conductuales afectados por un elevado estrés familiar [...] de forma significativa por cuestiones económicas, sociales o de salud [...]" (pág. 8).

Las secuelas y consecuencias del COVID-19 engloban aspectos físicos, emocionales, psicológicos y económicos, por los cuales se requiere un abordaje multidisciplinar, con factores que ayuden a aliviar las necesidades de la persona como: motivación, voluntarismo y optimismo tanto de recursos tangibles como intangibles; sin embargo cuando el tiempo ha transcurrido y la 
cotidianidad es una nueva realidad nueva y diferente muchas de las personas a las cuales les ha tocado afrontar dicha enfermedad han sufrido afectaciones en ámbitos tanto neurocognitivos y psicológicos.

Señalando a Balluerka, Gómez, Hidalgo, Gorostiaga, Espada, Padilla y Santed (2020b): los síntomas que se pueden presentar son falta de atención, pérdida de memoria, mala calidad de sueño, insomnio, ansiedad, depresión, estrés y fatiga, los mismos varían dependiendo de los factores relacionados, es decir, si hay casos de contagios o fallecidos dentro de su círculo cercano los síntomas serán de distinta intensidad debido a procesos de duelo o desencadenantes más particulares como sentimientos de vulnerabilidad, pesimismo, incertidumbre entre otros.

Desde las etapas iniciales la vulnerabilidad del personal de salud está presente debido a las emergencias sanitarias, las situaciones cómo lidiar con falta de insumos, medicación y camas, han sido factores que dicho personal ha experimentado lo cual generó impotencia y frustración, desde el inicio de la pandemia los mismos han estado expuestos al sufrimiento humano, jornadas extenuantes, recursos limitados, fatiga, estrés, ansiedad, tristeza, agotamiento físico y mental.

Sin embargo, los profesionales que elaboran en la Unidad de Cuidados Intensivos ( $\mathrm{UCl}$ ) son mayormente vulnerables para desarrollar el Síndrome de Burnout, debido a una mayor complejidad en el manejo y cuidado de los pacientes; es por eso la importancia de conocer en los ambientes laborales, no solo la prevalencia de este síndrome asociado al contexto del COVID-19 sino los factores que se encuentran inmersos.

Parafraseando a Urgilés (2020): refiere que existe la probabilidad de que el personal sanitario presente nuevos casos de depresión, ansiedad u otros trastornos psicológicos con mayor severidad, debido a la exposición de riesgo que experimentan en su entorno laboral, ya que pertenecer al personal de salud significa tener un gran nivel de responsabilidad, siendo el ambiente 
laboral algo influyente en la persona, es decir que el ambiente debería contribuir a la salud mental del personal.

La sobrecarga de trabajo y distintos factores generan un ambiente hostil, el cual amenaza el equilibrio psíquico y fisiológico del individuo, atentando contra el desempeño esperado del profesional. Es por lo que Peraza (2020): describe que a nivel mundial varios países han optado medidas como cuarentas, aislamientos, medidas de bioseguridad entre otros con el fin de mitigar la saturación de las $\mathrm{UCl}$ y emergencias del sistema de salud para que disminuya la carga del personal de salud.

Esta investigación tiene como objetivo general estimar los niveles de prevalencia del síndrome de Burnout en el personal de salud que atiende a pacientes con COVID-19, tomando en cuenta como objetivos específicos los factores de edad y sexo con mayor incidencia, relacionar la situación familiar de los participantes con dicho síndrome y reconocer la prevalencia en cada una de las dimensiones del síndrome.

\section{Metodología (Materiales y métodos)}

La presente investigación es de tipo cuantitativo, definida por Hernández, Fernández y Baptista (2014): como un enfoque en el que utiliza la recolección de datos para probar hipótesis con base en el análisis estadístico y la medición numérica. La investigación es descriptiva con una sola variable de diseño no experimental y tipo transversal, con una única recolección de datos.

Los participantes del estudio se eligieron bajo los siguientes criterios de inclusión: ser profesionales del área de salud, atender a pacientes con COVID19, laborar en la unidad de cuidados intermedios de una institución de salud, ser mayores de edad y consentir su participación en la investigación. Según estos criterios se utilizó el segmento completo del área una unidad de cuidados intermedios un hospital público de la ciudad de Quito, siendo la población total 
en estudio 40 profesionales a los que se les realizó: una encuesta de datos sociodemográficos y el inventario Maslach Burnout Inventory (MBI).

La encuesta de datos sociodemográficos contiene edad, sexo, estado civil, situación familiar y con quien convive en el hogar; con la finalidad de recopilar la mayor cantidad de datos de los participantes. Para ello, se aplicaron las normas éticas de trabajo con seres humanos, junto a los principios establecidos en las consideraciones éticas del informe de Belmont que hacen hincapié en criterios de respeto, beneficencia, voluntariedad y justicia.

El Inventario Maslach Burnout Inventory, muestra altos índices de consistencia interna y fiabilidad tanto en forma general de 0.80 y específicamente en cada dimensión de agotamiento emocional 0.90, despersonalización 0.79 y realización personal 0.71 , está constituido por 22 ítems en forma de afirmaciones, sobre los sentimientos y actitudes del profesional en su trabajo, cuyos rangos de medida se conforman por una escala Likert con medidas que parten desde: nunca (1), algunas veces al año (2), algunas veces al mes (3), algunas veces a la semana (4), diariamente (5). Así, se miden las tres dimensiones del síndrome de Burnout:

1. Agotamiento o cansancio emocional: valora la vivencia de estar exhausto emocionalmente como consecuencia de las demandas laborales, consta de nueve preguntas $(1,2,3,6,8,13,14,16,20)$, con una puntuación máxima de 45.

2. Despersonalización: valora el grado de reconocimiento de actitudes como frialdad, distanciamiento y ausencia de sentimientos hacia los demás, está compuesta por cinco ítems $(5,10,11,15,22)$, teniendo una puntuación máxima de 25.

3. Realización personal: evalúa sentimientos de auto eficiencia, competencia y realización personal exitosa en el trabajo, se compone de ocho ítems $(4,7,9,12,17,18,19,21)$, con una puntuación máxima de 40. 
Para el cálculo de cada uno de los ámbitos evaluados en el instrumento se suman las respuestas de los ítems dados en cada dimensión; con las puntuaciones máximas establecidas se tendrá el indicio de Burnout que va en el aspecto de agotamiento o cansancio emocional > 22, despersonalización > 7 y realización personal $<24$; tomando en cuenta y conociendo los valores 0 rangos.

Mencionando a Brito y Nelo (2017): la evaluación indicará indicios del síndrome de Burnout cuando existan altos valores en las dimensiones de agotamiento y despersonalización y bajos valores en realización personal. La clasificación y procesamiento de datos se realizó usando el programa estadístico informático Statistical Package for the Social Sciences (SPSS) el cual es un software de análisis estadísticos, en donde se sintetiza los resultados manejando en todo momento los altos niveles de confidencialidad que se indicaron en el consentimiento informado aceptado.

\section{Resultados}

A fin de tener una perspectiva clara y una caracterización inicial de los individuos de la muestra con la que se realizó esta investigación, se procesaron las encuestas sociodemográficas y el resumen de los resultados según las dimensiones con sus respectivas variables realizadas por las 40 personas que participaron en este estudio De dicho análisis se obtuvieron los datos recopilados.

Respecto a las variables nominales acerca de la población general de 40 profesionales de salud, se evidenció un $62,5 \%$ de mujeres y un $37,5 \%$ de hombres; por lo que existe predominancia de las mujeres. Entre los datos preponderantes de la muestra según el "rango de edad" se observa primeramente el rango de edad de 31 a 40 años ( $N=18$ y 45\%), siguiéndoles los rangos comprendidos de 41 a 50 años ( $N=8$ y 20\%), 21 a 30 años $(N=7$ y $17,5 \%), 51$ a 60 años ( $N=6$ y 15\%) y por último de 61 años a más ( $N=1$ y 2,5\%). 
Dentro de la variable "estado civil" sobresalen los individuos casados ( $\mathrm{N}=20$ y 50,0\%), continuando con los solteros ( $\mathrm{N}=10$ y $25,0 \%)$, unión libre $(\mathrm{N}=6$ y $15,0 \%$ ), separados ( $\mathrm{N}=3$ y $7,5 \%$ ), y finalmente los viudos ( $\mathrm{N}=1$ y $2,5 \%$ ). $\mathrm{La}$ variable "con quien vive" evidencio un elevado porcentaje en los individuos que viven con familia $(\mathrm{N}=32$ y $80,0 \%)$, por pareja $(\mathrm{N}=4$ y $10,0 \%)$, solo $(\mathrm{N}=4$ y $10,0 \%)$. Finalmente, en la variable relacionada "Tiene Hijos" se destacó con mayor frecuencia el $\mathrm{Si}(\mathrm{N}=32$ y $80,0 \%)$, y el $\mathrm{NO}(\mathrm{N}=8$ y $20,0 \%)$.

\subsection{Indicios de Burnout según factores de edad, sexo y situación familiar}

Conforme al sexo, las mujeres presentan mayor afectación por indicios del síndrome, ya que su relación es alta despersonalización con un $40,0 \%$, alto agotamiento $10,0 \%$ y baja realización con un $10,0 \%$ frente a los hombres con $17,5 \%-5,0 \%-2,5 \%$. Estos datos indican una fuerte tendencia hacia la afectación mayoritaria del síndrome sobre las mujeres.

El rango de edad comprendido entre 31 a 40 años, que es el de mayor peso en número de participantes ( $45 \%$ del total de la muestra), contiene el mayor número de individuos con indicios de presentar el síndrome, manteniendo una relación de alta despersonalización es de $61,1 \%$, alto agotamiento $11,1 \%$ y baja realización con porcentajes de $22,2 \%$ del total de individuos pertenecientes a este rango. Estos datos arrojan indicios que las edades entre 31 a 40 años son las más afectadas, además, al igual que con la variable sexo la despersonalización tiene un fuerte impacto en este caso en todos los rangos de edad ya que ninguno presenta un nivel bajo en esta dimensión.

De acuerdo con el estado civil, los individuos casados son los que presentan mayor afectación por indicios del síndrome ya que su relación de alta despersonalización es de $27,5 \%$, alto agotamiento emocional $7,5 \%$ y baja realización personal es del $10,0 \%$, el resto de los estados civiles tienen afectación preferencial en alguna dimensión, por ejemplo, los individuos en 
unión libre están mayormente afectados en la dimensión de agotamiento 5,0\%, los solteros en despersonalización 17,5\%.

Los participantes que viven con familia presentan mayor afectación por indicios de síndrome ya que su relación alta despersonalización es de $42,5 \%$, alto agotamiento $12,5 \%$ y baja realización es de 7,5\%. Estos datos indican una fuerte tendencia hacia la afectación mayoritaria del síndrome sobre los individuos con familia.

En la variable "tiene hijos", los participantes que tienen hijos presentan un mayor grado de afectación debido a los indicios del síndrome ya que su relación es de alta despersonalización con un $42,5 \%$, alto agotamiento $12,5 \%$ y baja realización de $12,5 \%$ frente a los participantes que no tienen hijos con una relación de alta despersonalización 15,0\% y alto agotamiento emocional $2,5 \%$, además los individuos que no tienen hijos se engloban en una alta realización personal 20,0\%.

Se desglosa la información en porcentajes sobre el indicio a presentar el Síndrome de Burnout en las distintas dimensiones con las variables de: sexo, edad y situación familiar (estado civil, con quien vive, tiene hijos); analizando los resultados mostrados en la tabla 1.

Tabla 1. Datos sociodemográficos y resultados de factores de estudio.

\begin{tabular}{|c|c|c|c|c|c|c|c|c|}
\hline \multicolumn{9}{|c|}{ DATOS SOCIODEMOGRÁFICOS GENERALES } \\
\hline $\begin{array}{r}\text { Rango } \\
\text { edad }\end{array}$ & $\begin{array}{c}21 \text { a } 30 \\
\text { (años) }\end{array}$ & \multicolumn{2}{|l|}{$\begin{array}{c}31 \text { a } 40 \\
\text { (años) }\end{array}$} & \multicolumn{2}{|c|}{$\begin{array}{c}41 \text { a } 50 \\
\text { (años) }\end{array}$} & $\begin{array}{c}51 \text { a } 60 \\
\text { (años) }\end{array}$ & $\begin{array}{c}\geq 61 \\
\text { (años) }\end{array}$ & Total \\
\hline Hombre & $1(2,5 \%)$ & \multicolumn{2}{|l|}{$5(12,5 \%)$} & \multicolumn{2}{|c|}{$5(12,5 \%)$} & $3(7.5 \%)$ & $1(2,5 \%)$ & $15(37,5 \%)$ \\
\hline Mujer & $6(15 \%)$ & \multicolumn{2}{|c|}{$13(32,5 \%)$} & \multicolumn{2}{|c|}{$3(7,5 \%)$} & $3(7.5 \%)$ & 0 & $25(62,5 \%)$ \\
\hline TOTAL & $7(17,5 \%)$ & \multicolumn{2}{|c|}{$18(45 \%)$} & \multicolumn{2}{|c|}{$8(20 \%)$} & $6(15 \%)$ & $1(2,5 \%)$ & $40(100 \%)$ \\
\hline \multicolumn{9}{|c|}{ SITUACIÓN FAMILIAR } \\
\hline \multicolumn{3}{|c|}{ Estado civil } & \multicolumn{4}{|c|}{ Con quien vive } & \multicolumn{2}{|c|}{ Tiene hijos } \\
\hline Soltero & \multicolumn{2}{|l|}{$10(25,0 \%)$} & \multicolumn{2}{|c|}{ Solo } & \multicolumn{2}{|c|}{$4(10,0 \%)$} & $\mathrm{Si}$ & $32(80,0 \%)$ \\
\hline Casado & \multicolumn{2}{|l|}{$20(50,0 \%)$} & \multicolumn{2}{|c|}{ Pareja } & \multicolumn{2}{|c|}{$4(10,0 \%)$} & No & $8(20,0 \%)$ \\
\hline Viudo & \multicolumn{2}{|l|}{$1(2,5 \%)$} & \multicolumn{2}{|c|}{ Familia } & \multicolumn{2}{|c|}{$32(80,0 \%)$} & & \\
\hline Unión libre & \multicolumn{2}{|l|}{$6(15,0 \%)$} & & & & & \\
\hline Separado & \multicolumn{2}{|l|}{$3(7,5 \%)$} & & & & & & \\
\hline \multicolumn{9}{|c|}{ RESUMEN DE RESULTADOS (\%) } \\
\hline
\end{tabular}


Instituto Internacional de Investigación y Desarrollo Tecnológico Educativo INDTEC, C.A.

DOI: https://doi.org/10.29394/Scientific.issn.2542-2987.2021.6.21.7.144-162

OAI-PMH: http://www.indteca.com/ojs/index.php/Revista Scientific/oai

Artículo Original / Original Article

\begin{tabular}{|c|c|c|c|c|c|c|c|c|c|}
\hline & \multicolumn{3}{|c|}{$\begin{array}{c}\text { Agotamiento } \\
\text { emocional }\end{array}$} & \multicolumn{2}{|c|}{ Despersonalización } & \multicolumn{3}{|c|}{ Realización personal } \\
\hline & & Bajo & Medio & Alto & Medio & Alto & Bajo & Medio & Alto \\
\hline \multirow{5}{*}{ 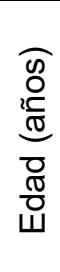 } & 21 a 30 & 42,9 & 42,9 & 14,3 & 14,3 & 85,7 & 0 & 0 & 100 \\
\hline & 31 a 40 & 50,0 & 38,9 & 11,1 & 38,9 & 61,1 & 22,2 & 16,7 & 61,1 \\
\hline & 41 a 50 & 50,0 & 37,5 & 12,5 & 62,5 & 37,5 & 12,5 & 25,0 & 62,5 \\
\hline & 51 a 60 & 66,7 & 16,7 & 16,7 & 66,7 & 33,3 & 0 & 16,7 & 83,3 \\
\hline & $\geq 61$ & 0 & 0 & 100 & 0 & 100 & 0 & 0 & 100 \\
\hline \multirow{2}{*}{ 爻 } & Mujer & 25,0 & 27,5 & 10,0 & 22,5 & 40,0 & 10,0 & 7,5 & 45,0 \\
\hline & Hombre & 25,0 & 7,5 & 5,0 & 20,0 & 17,5 & 2,5 & 7,5 & 27,5 \\
\hline \multirow{5}{*}{$\begin{array}{l}\bar{z} \\
\overline{0} \\
0 \\
0 \\
\frac{0}{0} \\
\bar{w} \\
w\end{array}$} & Soltero & 7,5 & 15,0 & 2,5 & 7,5 & 17,5 & 2,5 & 0 & 22,5 \\
\hline & Casado & 27,5 & 15,0 & 7,5 & 22,5 & 27,5 & 10,0 & 10,0 & 30,0 \\
\hline & Viudo & 2,5 & 0 & 0 & 2,5 & 0 & 0 & 0 & 2,5 \\
\hline & Unión libre & 5,0 & 5,0 & 5,0 & 5,0 & 10,0 & 0 & 2,5 & 12,5 \\
\hline & Separado & 7,5 & 0 & 0 & 5,0 & 2,5 & 0 & 2,5 & 5,0 \\
\hline \multirow{3}{*}{$\stackrel{\infty}{>}$} & Solo & 2,5 & 5,0 & 2,5 & 2,5 & 7,5 & 2,5 & 0 & 7,5 \\
\hline & Pareja & 7,5 & 2,5 & 0 & 2,5 & 7,5 & 2,5 & 2,5 & 5,0 \\
\hline & Familia & 40,0 & 27,5 & 12,5 & 37,5 & 42,5 & 7,5 & 12,5 & 60,0 \\
\hline \multirow{2}{*}{$\stackrel{\text { o }}{\frac{0}{1}}$} & $\mathrm{Si}$ & 42,5 & 25,0 & 12,5 & 37,5 & 42,5 & 12,5 & 15,0 & 52,5 \\
\hline & No & 7,5 & 10,0 & 2,5 & 5,0 & 15,0 & 0 & 0 & 20,0 \\
\hline
\end{tabular}

Fuente: Los Autores (2021).

\subsection{Prevalencia del síndrome de Burnout de la población de estudio}

Para el cálculo de cada uno de los ámbitos evaluados en el instrumento se suman las respuestas de los ítems dados en cada dimensión; con las puntuaciones máximas establecidas se tendrá el indicio de Burnout que va en el aspecto de agotamiento o cansancio emocional > 22, despersonalización > 7 y realización personal $<24$; tomando en cuenta y conociendo los valores y rangos.

Un análisis global y general de la muestra estudiada correspondiente a los 40 participantes encuestados que se presenta en dicho resumen es un estadístico general de los resultados para cada dimensión evaluada, que indica las medias, los mínimos y los máximos puntajes obtenidos en las 
distintas dimensiones y las respectivas desviaciones estándar e intervalo de confianza de IC 95\% respectivos.

Finalmente, la prevalencia de los participantes en las distintas dimensiones del Síndrome de Burnout es alta, debido a que de manera general se encuentran con indicios en una o dos dimensiones, sin embargo, ninguno de los participantes tiene indicios en las tres dimensiones; el $37,50 \%$ no presenta indicios de Burnout en ninguna dimensión, mientras que el 32,50\% presenta indicios en una dimensión, y el 30,00\% las presenta en al menos dos dimensiones, lo cual se puede visualizar en la tabla 2. Es importante mencionar que las variables asociadas como edad, sexo y situación familiar no son estadísticamente significativas lo cual se comprobó mediante la función del SPSS de chi-cuadrado $X^{2}$.

Tabla 2. Dimensiones en las que se evidencia indicios de Burnout.

\begin{tabular}{|l|c|c|c|c|}
\hline \multicolumn{3}{|c|}{ PUNTAJES DE REFERENCIA PARA DIMENSIONES DEL SÍNDROME DE BURNOUT } \\
\hline & Bajo & Medio & Alto & Indicios Burnout \\
\hline Agotamiento Emocional & $0-15$ & $16-22$ & $23-45$ & Más de 22 \\
\hline Despersonalización & $0-4$ & $5-7$ & $8-25$ & Más de 7 \\
\hline Realización Personal & $0-23$ & $24-28$ & $29-40$ & Menos de 24 \\
\hline
\end{tabular}

INFORME DE RESULTADOS DEL SÍNDROME DE BURNOUT Y SUS DIMENSIONES

\begin{tabular}{|c|c|c|c|c|}
\hline \multicolumn{2}{|c|}{ VARIABLES } & $\begin{array}{l}\text { Agotamiento } \\
\text { emocional }\end{array}$ & Despersonalización & $\begin{array}{l}\text { Realización } \\
\text { personal }\end{array}$ \\
\hline \multicolumn{2}{|l|}{$\mathrm{N}$} & 40 & 40 & 40 \\
\hline \multicolumn{2}{|l|}{ IC $95 \%$} & $14,66-17,83$ & $7,54-9,30$ & $28,26-32,98$ \\
\hline \multicolumn{2}{|l|}{ Mínimo } & 8 & 5 & 8 \\
\hline \multicolumn{2}{|l|}{ Máximo } & 29 & 17 & 38 \\
\hline \multicolumn{2}{|l|}{ Media } & 16,25 & 8,42 & 30,62 \\
\hline \multicolumn{2}{|c|}{ Desviación } & 4,94 & 2,75 & 7,38 \\
\hline \multirow{3}{*}{$\%$ Nivel } & Nivel Alto & $15 \%$ & $57,5 \%$ & $72,5 \%$ \\
\hline & Nivel Medio & $35 \%$ & $42,5 \%$ & $15 \%$ \\
\hline & Nivel Bajo & $50 \%$ & $0 \%$ & $12,5 \%$ \\
\hline \multicolumn{5}{|c|}{ DIMENSIONES EN LAS QUE SE EVIDENCIA SÍNDROME DE BURNOUT } \\
\hline & & & Número & $\%$ \\
\hline \multicolumn{2}{|c|}{$\begin{array}{l}\text { Presenta Burnout en tres dimensiones } \\
\text { No presenta Burnout en ninguna dimensión }\end{array}$} & $\begin{array}{l}\text { sión } \\
\text { siones } \\
\text { ensiones } \\
\text { una dimensión }\end{array}$ & $\begin{array}{c}13 \\
12 \\
0 \\
15\end{array}$ & $\begin{array}{c}32,50 \\
30,00 \\
0,00 \\
37,50\end{array}$ \\
\hline \multicolumn{3}{|c|}{$\begin{array}{c}\text { No presenta Burnout en ninguna dimensión } \\
\text { TOTAL }\end{array}$} & 40 & 100 \\
\hline
\end{tabular}

Fuente: Los Autores (2021). 


\section{Conclusiones}

La mayoría de participantes presentan indicios de burnout en una o dos dimensiones de $32,5 \%$ y $30 \%$, por lo que sumadas se establece de manera general un $62,5 \%$ de la muestra que padecen dichos indicios; sin embargo, existe prevalencia en niveles altos de despersonalización ya que el 57,5\% la presenta; los cuales son propensos a actitudes de frialdad, distanciamiento, ausencia de sentimientos hacia los demás; en cuanto a un nivel alto de agotamiento un $15 \%$ sufren esta afectación, caracterizándose por actitudes y sensaciones negativas sobre los demás y valoran la vivencia de estar exhausto emocionalmente por las demandas laborales a causa del COVID-19.

La dimensión menos afectada es la realización personal en nivel bajo con apenas un $12,5 \%$ de los participantes, mostrando que el personal de salud no siente que tenga afectación en cuanto a su desempeño y competencias al momento de realizar su trabajo a pesar del aumento en la exigencia laboral que la pandemia del COVID-19 ha traído.

El personal sanitario con relación a las variables de edad, sexo y situación familiar que presenta mayor indicio en presencia del síndrome de Burnout en una o dos dimensiones son: las mujeres en la relación alto agotamiento $10 \%$, alta despersonalización $40 \%$ y baja realización $10 \%$, por lo que sumando estos porcentajes un $60 \%$ de mujeres está afectada.

Los rangos de edades de la muestra en las cuales existe mayor incidencia son individuos entre 31 a 40 años; en cuanto a la situación familiar se concluye que los participantes que son casados $50,0 \%$, que viven con familia $80,0 \%$ y que tienen hijos $80,0 \%$ se encuentran con mayor afectación o son más propensos a desarrollar indicios de Burnout.

\section{Referencias}

Álvarez, A. (2020). La Historia del COVID 19 en tiempos del Coronavirus.

Un ensayo inconcluso. Pasado Abierto, 11, 215-235 e-ISSN: 2451- 
6961. Recuperado de: https://ri.conicet.gov.ar/handle/11336/114881 Balluerka, N., Gómez, J., Hidalgo, M., Gorostiaga, A., Espada, J., Padilla, J., \& Santed, G. (2020a,b). Las consecuencias psicológicas de la COVID19 y el confinamiento. Informe de investigación. España: Servicio de Publicaciones de la Universidad del País Vasco. Recuperado de: http://hdl.handle.net/10810/45924

Brito, X., \& Nelo, J. (2017). Elaboración de una propuesta de un programa de seguridad $y$ salud en el trabajo en una empresa de telecomunicaciones ubicada en el área metropolitana de Caracas, Venezuela. Venezuela: Universidad Católica Andrés Bello.

Hernández, R., Fernández, C., \& Baptista, M. (2014). Metodología de la Investigación. Sexta edición, ISBN: 978-1-4562-2396-0. México: McGraw-Hill / Interamericana Editores, S.A. de C.V.

Juárez, A. (2020). Síndrome de burnout en personal de salud durante la pandemia COVID-19: un semáforo naranja en la salud mental. Salud UIS, 52(4), 432-439, e-ISSN: 2145-8464. Recuperado de:

https://revistas.uis.edu.co/index.php/revistasaluduis/article/view/11528 Martínez, A. (2010). El Síndrome de Burnout. Evolución conceptual y estado actual de la cuestión. Vivat Academia, (112), 42-80, e-ISSN: 1575-2844. Recuperado de:

https://www.redalyc.org/articulo.oa?id=525752962004

Maslach, C., \& Jackson, S. (1981). The measurement of experienced burnout. Journal of Organizational Behavior, 2, 99-113, e-ISSN: 08943796. Recovered from: https://doi.org/10.1002/job.4030020205

Maslach, C., Jackson, S., \& Leiter, M. (1996). Maslach burnout Inventory. Third edition. Palo Alto, CA., United States: Consulting Psychologists Press.

OMS (2020). COVID-19: cronología de la actuación de la OMS. Ginebra, Suiza: Organización Mundial de la salud. 
Peraza, C. (2020). Salud laboral frente a la pandemia del COVID-19 en Ecuador. MediSur, 18(3), 507-511, e-ISSN: 1727-897X. Recuperado de: http://medisur.sld.cu/index.php/medisur/article/view/4713

UGT-Andalucía (2009). Guía de prevención de riesgos psicosociales en el trabajo. D.L.: SE 5203-2009. Andalucía, España: Departamento de Asistencia Técnica para la Prevención de Riesgos Laborales UGT Andalucía; Fundación para la Prevención de Riesgos Laborales.

Urgilés, S. (2020). Síndrome de burnout en el personal de salud que trabaja en la unidad de terapia intensiva durante la pandemia COVID-19 en un hospital de Quito. Tesis. Ecuador: Universidad Internacional SEK. Recuperado de:

http://repositorio.uisek.edu.ec/handle/123456789/3970

Velázquez, L. (2020). La COVID-19: reto para la ciencia mundial. Anales de la Academia de Ciencias de Cuba, 10(2), 1-2, e-ISSN: 2304-0106. Recuperado de:

http://revistaccuba.sld.cu/index.php/revacc/article/view/763 


\section{Edwin Guillermo Valencia González \\ e-mail: edwinvalencia@uti.edu.ec}

Nacido en Quito, Ecuador, el 14 de octubre del año

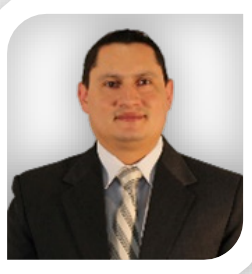
1980. Máster en Desarrollo del Talento Humano por la Universidad Andina Simón Bolívar (UASB); Especialista Superior en Dirección de Personas y del Talento Humano por la Universidad Andina Simón Bolívar; Psicólogo Industrial por la Universidad Central del Ecuador (UCE), Diplomado en Docencia Universitaria e investigación por el Centro Universitario (CIFE), México; he ejercido la docencia por más de cinco años en instituciones de educación superior del Ecuador, en temáticas afines a la Gestión del Talento Humano; también he desempeñado labores directivas en el campo del manejo del talento humano en instituciones públicas y privadas, desarrollando equipos de trabajo de alto desempeño por más de 14 años; actualmente dedicado a la docencia a tiempo completo en la Universidad Tecnológica Indoamérica (UTI); coautor del libro titulado Seleccionar Directivos en Centros Educativos 2020. 


\section{Daniela Alejandra Correa Sinche}

e-mail: danny ale0202@hotmail.com

Nacida en Quito, Ecuador, el 26 de junio del año 1998. He

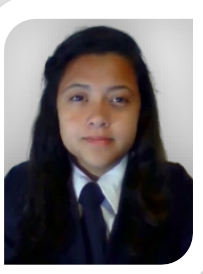

participado en varios congresos, seminarios y talleres enfocados a la formación académica en relación con aspectos psicológicos adquiriendo conocimientos y experiencia; de igual forma he desempeñado labores en la Organización Children International; también he colaborado en ámbitos de catequesis y desarrollo de habilidades sociales con estudiantes de secundaria por más de cinco años; mis intereses están centrados en el ámbito de la investigación científica de diversos aspectos sociales y psicológicos. 


\section{Vanesa Katherine Pazmiño Cruz \\ e-mail: vanepazzmi1998@hotmail.es}

Nacida en Quito, Ecuador, el 1 de abril del año 1998. He

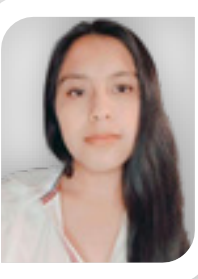
desempeñado labores en la Organización Children International; también he puesto en práctica todos mis conocimientos y he ido adquiriendo experiencia, con el fin de crecer personal y profesionalmente; cursando seminarios sobre Jornadas de la nueva Psicología; Congreso de Vinculación con la Sociedad; Sensibilización en Discapacidades; Prevención Integral del Uso y Consumo de Drogas en el Ámbito de Educación Superior; actualmente egresada de la Facultad de Ciencias Humanas y de la Salud. 